\title{
Extracting Structure from Contaminated Symbolic Data
}

\author{
Antony Pearson \\ antony.pearson@colorado.edu \\ University of Colorado \\ Boulder, Colorado
}

\begin{abstract}
Symbolic data is the epitome of modern biological datasets. Modern sequencing technologies produce millions of reads giving insights on genome sequence, transcription levels, epigenetic modifications, and much more. To analyze those sequences one usually makes assumptions on their underlying structure, e.g., that the number of reads has Poisson distribution, or that transcription factor binding events occur independently at nonoverlapping promoters. These types of assumptions are often not exactly correct in reality. In fact, even when they are valid, a small amount of data "contamination" may make them appear untrue. The traditional approach to questioning assumptions on data has been hypothesis testing. This approach has various shortcomings, however. Particularly, its Boolean nature does not give room for a null hypothesis to be "approximately true." This tutorial introduces a methodology to assess statistical assumptions on symbolic data that may be contaminated. It will give a general overview on how to approach these problems numerically, and present analytical results for some special classes of structured probability distributions. It will demonstrate the applicability of this rather new methodology with DNA methylation data to question the common but unconscious assumption that methylation of CpGs is exchangeable, and transcription factor binding site $k$-mers to question the use of logoplots in summarizing TFBS behaviour. Data and code for this tutorial, in the form an iPython Notebook, will be made available via GitHub. This work is in collaboration with M. E. Lladser.
\end{abstract}

\section{KEYWORDS}

symbolic data, contamination, exchangeability, epigenetics, DNA methylation, independence, transcription factor binding sites

\section{ACM Reference Format:}

Antony Pearson. 2019. Extracting Structure from Contaminated Symbolic Data. In 10th ACM International Conference on Bioinformatics, Computational Biology and Health Informatics (ACM-BCB '19), September 7-10, 2019, Niagara Falls, NY, USA. ACM, New York, NY, USA, 1 page. https://doi.org/ $10.1145 / 3307339.3343175$

\section{SUMMARY}

Models describing contamination of continuous data have been well-studied and date back to the 19th century [3]. Such models

Permission to make digital or hard copies of part or all of this work for personal or classroom use is granted without fee provided that copies are not made or distributed for profit or commercial advantage and that copies bear this notice and the full citation on the first page. Copyrights for third-party components of this work must be honored For all other uses, contact the owner/author(s).

ACM-BCB '19, September 7-10, 2019, Niagara Falls, NY, USA

(C) 2019 Copyright held by the owner/author(s).

ACM ISBN 978-1-4503-6666-3/19/09.

https://doi.org/10.1145/3307339.3343175 typically assume that there is a true underlying probabilistic process producing data, and that with some small probability $\epsilon$ a sample is drawn from an alternate, or contaminating distribution. Then the probabilistic source producing observation can be described as a mixture model [1] :

$$
P=(1-\epsilon) \cdot P_{0}+\epsilon \cdot R,
$$

where $P_{0}$ represents the underlying process. In continuous settings, certain restrictions on the component $R$ (such as following a Gaussian law with mean identical to $\left.P_{0}\right)$ are required for inference and estimation [4].

In symbolic settings, such as when data are sequencing reads, restrictions on the structure of the contamination have meaningful implications on our beliefs about the probabilistic source. Instead, we use the intuitive mixture representations above and find the smallest $\epsilon \geq 0$, with $R$ an arbitrary probabilistic model, so that $P_{0}$ has certain desired structure. Unlike a $p$-value, which is highly dependent on sample size, this quantity depends only on the underlying source $P$ and describes the fraction of data that can be expected to follow the desired structure. We present numerical methodology for approximating the amount of contamination and derive explicit expressions for this quantity in some special cases.

We demonstrate applicability of the methodology above by identifying loci where the assumption that DNA methylation is exchangeable is violated. We also identify transcription factor binding sites whose sequences are particularly poorly-described by a logoplot, because the assumption that DNA bases are independent is badly violated [2].

\section{ACKNOWLEDGMENTS}

This material is based upon work supported by the National Science Foundation Graduate Research Fellowship under Grant No. (2016198773) and partially funded by the NSF IGERT 1144807 grant.

\section{REFERENCES}

[1] Peter J. Huber. 1964. Robust Estimation of a Location Parameter. Ann. Math. Statist. 35, 1 (03 1964), 73-101. https://doi.org/10.1214/aoms/1177703732

[2] Aziz Khan, Oriol Fornes, Arnaud Stigliani, Marius Gheorghe, Jaime A CastroMondragon, Robin van der Lee, Adrien Bessy, Jeanne Chèneby, Shubhada $\mathrm{R}$ Kulkarni, Ge Tan, Damir Baranasic, David J Arenillas, Albin Sandelin, Klaas Vandepoele, Boris Lenhard, Benoît Ballester, Wyeth W Wasserman, François Parcy, and Anthony Mathelier. 2017. JASPAR 2018: update of the open-access database of transcription factor binding profiles and its web framework. Nucleic Acids Research 46, D1 (11 2017), D260-D266. https://doi.org/10.1093/nar/gkx1126 arXiv:http://oup.prod.sis.lan/nar/article-pdf/46/D1/D260/23162408/gkx1126.pdf

[3] S. Newcomb. 1882. Discussion and results of observations on transits of Mercury from 1677 to 1881. [United States. Nautical Almanac Office. Astronomical paper ; v.1, pt. 6 (1882)], [Washington : U.S. Nautical Almanac Office, 1882], p. 363-487 : charts ; $29 \mathrm{~cm} .1$ (1882), 363-487.

[4] Antonio Punzo and Paul D. McNicholas. 2016. Parsimonious mixtures of multivariate contaminated normal distributions. Biometrical fournal 58, 6 (2016), 1506-1537. https://doi.org/10.1002/bimj.201500144 arXiv:https://onlinelibrary.wiley.com/doi/pdf/10.1002/bimj.201500144 